\title{
A Study of Ischiopubic Index Using X-Ray Films in Lagos State of Nigeria
}

\author{
Theresa B. Ekanem, Ekaette J. Akpan, and Otu E. Mesembe \\ Department of Anatomy, Faculty of Basic Medical Sciences, University of Calabar, PMB 1115, Calabar, Cross River State, Nigeria \\ Correspondence should be addressed to Otu E. Mesembe; drotumesembe@gmail.com
}

Received 26 May 2014; Accepted 9 September 2014; Published 25 September 2014

Academic Editor: Carsten Lippold

Copyright (C) 2014 Theresa B. Ekanem et al. This is an open access article distributed under the Creative Commons Attribution License, which permits unrestricted use, distribution, and reproduction in any medium, provided the original work is properly cited.

\begin{abstract}
The ischiopubic index was studied in adult pelvic X-ray films of subjects aged eighteen to seventy years. A total of $120 \mathrm{X}$-ray films made of 60 males and 60 females were collected from LUTH, Lagos State. The ischiopubic index was calculated by dividing the pubic length by ischial length and by multiplying by 100. The mean ischiopubic index for males was $101.05 \pm 16.65$ and that of the females was $115.99 \pm 18.5$. Sex differences of these indices were statistically significant $(P<0.001)$ for both sexes. The mean length of the females pubis was significantly longer than that for males $(P<0.001)$, and similarly the mean length of ischium in males was significantly longer than that of the females $(P<0.001)$. Using $\mathrm{X}$-ray films, sex could be assigned using demarking points to $47 \%$ and 50\% females in Lagos State. In conclusion, this research has provided evidence that the ischiopubic index and the demarking points are reliable in sexing the hip bones of Nigerians (using X-ray films). Thus the ischiopubic index may be of value in forensic and archaeological analyses and in solving medicolegal cases.
\end{abstract}

\section{Introduction}

The innominate bone has long been recognised as one of the best skeletal indicators of sex in an adult individual. Methods have been developed which provide criteria for sex determination of adults using features of the innominate bone $[1,2]$. Various studies have shown extensively that the hip bone is an ideal bone for sex determination because it reflects not only the general differences between the sexes but also the special adaptation of the female hip bone for child bearing [35].

lbone of females was much longer than those of the males. This has been confirmed in adult black Malawians [5]. In the past many workers have evolved various metrical parameters and indices for sexing of the hip bone of which one of them is the ischiopubic index. This index was used efficiently where the pubic was separated and could determine sex in a high percentage of bones. The ischiopubic index is useful in sex differentiation [2]. It is obtained by dividing the length of pubis by the length of the ischium. This index is one of the determining factors of the size of the pelvic inlet and is significantly higher in females than in males [4].
The results obtained will contribute to comparative data base for Nigerians [6] as well as compare those of other races.

\section{Materials and Methods}

A total of 120 radiographs of the anterior-posterior view of the pelvis were collected comprising 60 males and 60 females. All the radiographs were collected from the University of Lagos Teaching Hospital (LUTH), Lagos State, Nigeria. Only normal adult radiographs of subjects aged between 18 and 70 years were used.

Materials included the $\mathrm{X}$-ray illuminator, the radiographic films, a pencil, and transparent metric rule.

2.1. Measurement Technique. The technique used for measuring the ischiopubic index was described by Washburn [2] and Schult and Adolf [7]. It was determined by dividing the length of the pubis by the length of ischium and multiplied by hundred. The length of the pubis and ischium was measured from the point where they meet the acetabulum. In taking these measurements, X-ray films were placed on 
TABLE 1: Measurement in mm of pubic length, ischial length, and ischiopubic index in males in Lagos State.

\begin{tabular}{lccc}
\hline Measurement and calculations & Pubic length & Ischial length & Ischiopubic index \\
\hline Number & 60 & 60 & 60 \\
Mean & $68.7^{*}$ & $68.93^{*}$ & $101.05^{*}$ \\
Standard deviation & \pm 9.62 & \pm 10.77 & \pm 16.65 \\
Actual range & $43-85$ & $50-90$ & $75-133.33$ \\
Identification point & $<58$ & $>90.00$ & $<92.05$ \\
Percentage identification point & $10 \%$ & $0 \%$ & $23.33 \%$ \\
Calculated range & $59.11-78.35$ & $58.16-79.7$ & $84.4-117.7$ \\
Demarking point & $<68.12$ & $>75.44$ & $<97.49$ \\
Percentage demarking point & $43.33 \%$ & $26.67 \%$ & 46.67 \\
\hline
\end{tabular}

${ }^{*} P<0.001$ was statistically significant (male versus female).

the horizontal surface of an illuminator. The drawing shown in Figure 1 below illustrates the technique and the landmark used in arriving at an accurate measurement of the length of the pubis and ischium.

Length of Ischium. This is a straight line AC drawn from the centre of the acetabulum rim to the most lower point on inferior border of ischial tuberosity.

Length of Pubis. This is a straight line $\mathrm{AB}$ drawn from the centre of the acetabulum to the medial end of the pubis:

$$
\text { Ischiopubic index: } \frac{\text { Length of Pubis }}{\text { Length of Ischium }} \times 100 \text {. }
$$

2.2. Statistical Analysis and Calculations. The data obtained from the measured parameters were analyzed statistically using one-way analysis of variance (ANOVA). The Student's $t$-test was applied to determine the sex differences. Values of $P<0.001$ were taken as being significant.

The actual range of the index for the male and female sexes was found out and the values were used in sex determination. The identification points were taken from the actual range for both sexes. The parameters were then subjected to demarking points (DPs) analysis as evolved by Jit and Singh [8].

\section{Results}

The values of the data obtained from the X-ray measurements of the pubic and ischial lengths of the hip bone and their ischiopubic indices for the 120 radiographs are shown in Tables 1,2, and 3. The pubic length in females was observed to be higher than those of the males, while the ischial length of males was more than that of the females.

3.1. Pubic Length. The pubic length of females was noticed to be more than that of the males. In Tables 1 and 2, the pubic length of the male hipbone varied from $43 \mathrm{~mm}$ to $83 \mathrm{~mm}$ with a mean value of $68.7 \mathrm{~mm} \pm 9.62$, while the pubic length of the female hipbone varied from $58 \mathrm{~mm}$ to $91 \mathrm{~mm}$ with a mean value of $76.6 \mathrm{~mm} \pm 8.48$. No female pubic length was less than $58 \mathrm{~mm}$; thus any pubic length less than this was classified as

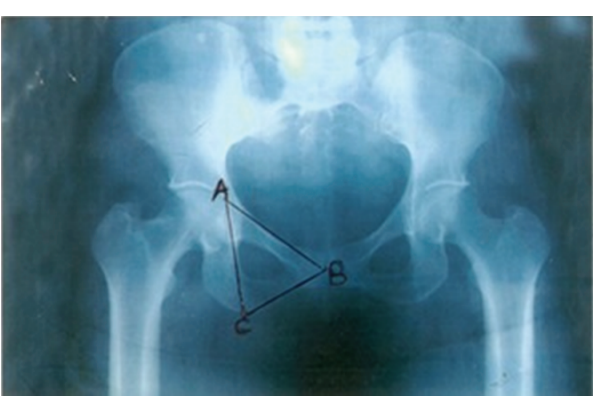

FIGURE 1: The radiograph of pelvis showing the measurements of the ischial length $\mathrm{AC}$ and pubic length $\mathrm{AB}$ (Ekanem et al. [6]).

male. Hence $58 \mathrm{~mm}$ serves as identification point (I.P) for the male hipbone. Likewise, no male pubic length was greater than $85 \mathrm{~mm}$; thus any male pubic length greater than this was classified as female. Hence the maximum pubic length for male served as I.P for female hipbone. Such identification point assigned sex to about $10 \%$ males and $16.67 \%$ females. The percentage beyond demarking point was $43.33 \%$ for both sexes.

The demarking point as seen in Tables 1 and 2 , shows that the male pubic length was less than $68.12 \mathrm{~mm}$ while that of the female was greater than $78.35 \mathrm{~mm}$.

3.2. Ischial Length. The ischial length of males was higher than that of the females. The ischial length of the female hipbone varied from $55 \mathrm{~mm}$ to $99 \mathrm{~mm}$ with a mean value of $66.97 \pm 8.47$, while that of the male varied from $50 \mathrm{~mm}$ to $90 \mathrm{~mm}$ with a mean value of $68.93 \pm 10.77$. The maximum ischial length of the females $90 \mathrm{~mm}$ served as I.P for males and the lowest ischial length for males $50 \mathrm{~mm}$ served as I.P for females. Such identification point assigned $0 \%$ for both sexes. As seen in Tables 1 and 2 the demarking point for male was greater than $75.44 \mathrm{~mm}$; therefore, any ischial length less than $58.16 \mathrm{~mm}$ was bound to be female. The percentage identified by demarking point was $26.67 \%$ for males and $13.33 \%$ for females.

3.3. Ischiopubic Index. The ischiopubic index of the male hipbone as seen in Table 1 varies from 75 to 133.33 with a mean 
TABLE 2: Measurement in mm of pubic length, ischial length, and ischiopubic index in females in Lagos State.

\begin{tabular}{lccc}
\hline Measurement and calculations & Pubic length & Ischial length & Ischiopubic index \\
\hline Number & 60 & 60 & 60 \\
Mean & $76.6^{*}$ & $66.97^{*}$ & $11.5 .99^{*}$ \\
Standard deviation & \pm 8.48 & \pm 8.47 & \pm 18.5 \\
Actual range & $58-91$ & $55-90$ & $92.05-163.63$ \\
Identification point & $>85$ & $<50$ & $>133.33$ \\
Percentage identification point & $16.67 \%$ & $0 \%$ & $13.33 \%$ \\
Calculated range & $68.12-85.08$ & $58.5-75.44$ & $97.49-134.49$ \\
Demarking point & $>78.35$ & $<58.16$ & $<117.7$ \\
Percentage demarking point & $43.33 \%$ & $13.33 \%$ & $50 \%$ \\
\hline
\end{tabular}

${ }^{*} P<0.001$ was statistically significant (male versus female).

TABLE 3: Comparative analysis of pubic length, ischial length, and ischopubic index in different races.

\begin{tabular}{|c|c|c|c|c|c|c|c|c|c|c|c|c|}
\hline \multirow{3}{*}{ Racial group source } & \multicolumn{4}{|c|}{ Pubic length } & \multicolumn{4}{|c|}{ Ischial length } & \multicolumn{4}{|c|}{ Ischiopubic index } \\
\hline & \multicolumn{2}{|c|}{ Male } & \multicolumn{2}{|c|}{ Female } & \multicolumn{2}{|c|}{ Male } & \multicolumn{2}{|c|}{ Female } & \multicolumn{2}{|c|}{ Male } & \multicolumn{2}{|c|}{ Female } \\
\hline & Number & Mean & Number & Mean & Number & Mean & Number & Mean & Number & Mean & Number & Mean \\
\hline Eskimos [4] & 129 & 74.1 & 95 & 80.1 & 129 & 88.4 & 95 & 81.0 & 129 & 83.9 & 95 & 98.8 \\
\hline American Whites [1] & 100 & 73.8 & 100 & 77.9 & 100 & 88.4 & 100 & 78.3 & 100 & 83.6 & 100 & 99.5 \\
\hline American Negros [1] & 50 & 69.2 & 50 & 73.5 & 50 & 86.6 & 50 & 77.5 & 50 & 79.9 & 50 & 95.0 \\
\hline Bantus $[1,2]$ & 82 & 66.2 & 70 & 73.2 & 82 & 80.3 & 70 & 74.8 & 82 & 82.5 & 70 & 78.1 \\
\hline Australian Aborigines [4] & 89 & 63.3 & 72 & 69.2 & 89 & 81.2 & 72 & 74.7 & 89 & 76.0 & 72 & 92.7 \\
\hline Nigerians [6] & 114 & 65.8 & 100 & 75.6 & 114 & 70.1 & 100 & 64.5 & 114 & 94.2 & 100 & 117.3 \\
\hline Nigerians (present study) & 60 & 68.7 & 60 & 76.6 & 60 & 68.93 & 60 & 66.97 & 60 & 101.05 & 60 & 115.99 \\
\hline
\end{tabular}

value of $101.05 \pm 16.65$, while that of the female hipbone as seen in Table 2 varies from 92.05 to 163.63 with a mean value of $115.99 \pm 18.5$. The maximum ischiopubic index for male, 133.33, served as identification point for the females and the lowest ischiopubic index for females 95.05 served as I.P for males. Such identification point assigned sex to about $23.33 \%$ of male hipbone and $13.33 \%$ of female hipbone.

As seen in Tables 1 and 2, any ischiopubic index less than 97.49 was bound to be male and any ischiopubic index greater than 117.7 was bound to be female. This index was higher in females than in males. Percentage beyond demarking point was $46.67 \%$ for males and $50 \%$ for females.

\section{Discussion}

The distinctive morphology of the human bone (innominate) and its clear sexual dimorphism makes it of interest from anatomical, anthropological, and forensic point of view. The authors who have studied this bone have paid attention either to features relating to its total size or to these of various components such as its inferior border, greater sciatic notch, the symphyseal surface, the acetabulum, the obturator foramen, and the arcuate line [1-3, 9].

The male pelvis is heavier and thicker than the female pelvis and usually has more prominent bony markings. The female pelvis is wider, shallower and has a larger pelvic inlet and outlet. Anatomical differences in the human pelvis are more pronounced during pubertal age especially in females. It is believed that sex hormones have a part to play in this variation. In females, the secretion of these hormones increases with age and reaches its peak during adolescence, while in males it was found out that the shape of the innominate bone is transformed to the male type under the influence of androgen [10].

In the present study, the pubic and ischial length could not identify reasonable percentage of pelvis in both males and females though the mean values of these parameters were statistically significant $(P<0.001)$ when they were compared. They could not be used in accurate sexing because the percentage identified by demarking points was low. However, the ischiopubic index was observed to be useful in sex differentiation.

The mean values of ischiopubic index were observed to be statistically significant $(P<0.001)$ and the percentage identified using demarking points was $47 \%$ for males and $50 \%$ for females in Lagos State. Washburn $[1,2]$ working on this index identified $80 \%$ and $100 \%$ American males and females, respectively. The slight low percentage of demarking point in the present study compared with the work of Washburn [1] may be due to the small number of X-ray films used.

Igbigbi and Msamati [5] measured the ischiopubic index in black Malawians and found that it was useful in sex determination using adult skeletons or X-ray film. With skeletal bones, sex was assigned to $92.3 \%$ males and females while X-ray films assigned sex to $87.8 \%$ males and $100 \%$ females. Also in a study conducted in Cross River State in Nigeria, Ekanem et al. [6] measured this index and the percentage identified by demarking points was $69 \%$ for males and $81 \%$ for females. 
In the present study, the mean pubic length of males was recorded as $68.73 \mathrm{~mm}$ for Lagos State while that of the females was $76.66 \mathrm{~mm}$. The ischial length for Lagos State residents was recorded as $68.93 \mathrm{~mm}$ and that of the females was $66.97 \mathrm{~mm}$. These were the lowest when compared to those of other races. For the males, the American Whites and Eskimos had the highest value of $89.4 \mathrm{~mm}$ while for the females the Eskimos had the highest ischial length of $81.0 \mathrm{~mm}$ followed by American Whites with a value of $78.3 \mathrm{~mm}[1,4]$. In Lagos State the mean ischiopubic index was 101.05 for males and 115.99 for females; when this was compared with the data from Cross River State [6], it was rated low.

Population differences which have been observed may be due to environmental or hereditary factors. According to MacLaughlin and Bruce [11], the pubic length for both sexes particularly that of the female showed accelerated changes with body size. Body size is known to be influenced by a particular environment; for instance, White children tend to mature faster than Black children and growth depends on skeletal development. Thus environmental as well as hereditary factors may influence racial differences.

The sex differences in the pubic and ischial lengths were found to be statistically significant when male and female data were compared $(P<0.001)$. Their mean ischiopubic indices were found to be statistically significant. The demarking points of these parameters were worked out to determine sex. The demarking point for the ischiopubic index was more useful in sex determination assigning sex to $47 \%$ male and $50 \%$ females in Lagos State. Important findings included the fact that there were significant differences between the male and female ischiopubic indices. Therefore the ischiopubic index is a useful parameter in sexing of hip bone.

In conclusion, this research has provided evidence that the ischiopubic index and the demarking points are reliable in sexing the hip bones of Nigerians (using X-ray films). Thus the ischiopubic index may be of value in forensic and archaeological analyses and in solving medicolegal cases.

\section{Conflict of Interests}

The authors declare that there is no conflict of interests regarding the publication of this paper.

\section{References}

[1] S. L. Washburn, "Sex differences in the pubic bone," American Journal of Physical Anthropology, vol. 6, no. 2, pp. 199-207, 1948.

[2] S. L. Washburn, "Sex differences in the pubic bone of Bantu and Bushman," The American Journal of Physical Anthropology, vol. 7, no. 3, pp. 425-432, 1949.

[3] F. P. Schulter-Ellis, D. J. Schmidt, L. A. Hayek, and J. Craig, "Determination of sex with a discriminant analysis of new pelvic bone measurements-part I," Journal of Forensic Sciences, vol. 28, no. 1, pp. 169-180, 1983.

[4] V. Davivong, "The pelvic girdle of the Australian Aborigine; sex differences and sex determination," The American Journal of Physical Anthropology, vol. 21, pp. 444-455, 1963.
[5] P. S. Igbigbi and B. C. Msamati, "Ischio-pubic index in adult black Malawians," East African Medical Journal, vol. 77, no. 9, pp. 514-516, 2000.

[6] T. B. Ekanem, A. J. Udongwu, and S. P. Singh, "Radiographic determination of sex differences in ischiopubic index of a Nigerian population," The Internet Journal of Biological Anthropology, vol. 3, no. 2, 2009.

[7] A. H. Schult and H. Adolf, "Sex differences in the pelvis of primate," American Journal of Physical Anthropology, vol. 8, pp. 401-424, 1950.

[8] I. Jit and S. Singh, "The sexing of the adult clavicles," The Indian Journal of Medical Research, vol. 54, no. 6, pp. 551-571, 1966.

[9] S. Singh and B. R. Potturi, "Greater sciatic notch in sex determination," Journal of Anatomy, vol. 125, no. 3, pp. 619-624, 1978.

[10] T. Iguchi, S. Irisawa, Y. Fukazawa, Y. Uesugi, and N. Takasugi, "Morphometric analysis of the development of sexual dimorphism of the mouse pelvis," Anatomical Record, vol. 224, no. 4, pp. 490-494, 1989.

[11] S. M. MacLaughlin and M. F. Bruce, "Population variation in sexual dimorphism in the human innominate," Human Evolution, vol. 1, no. 3, pp. 221-231, 1986. 

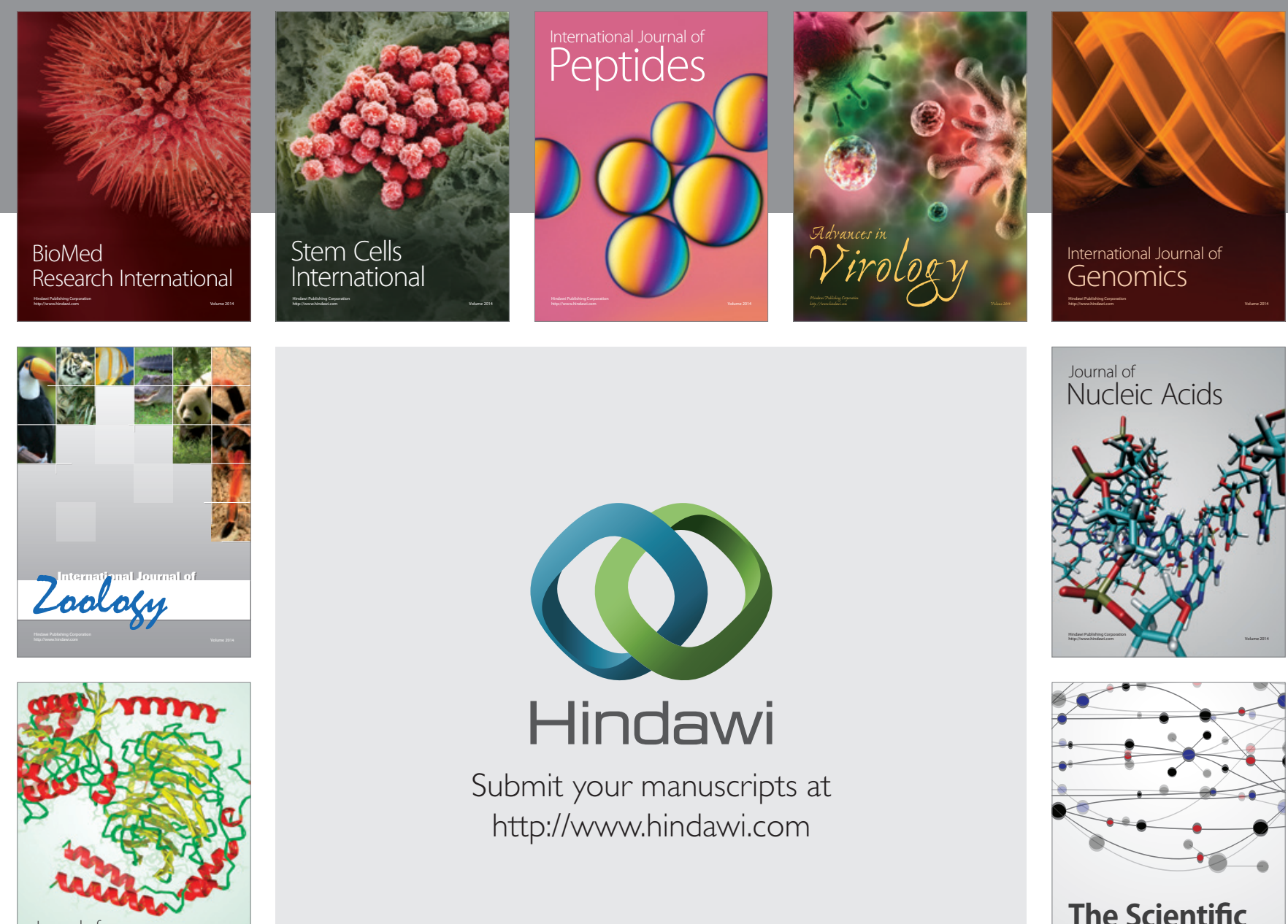

Submit your manuscripts at

http://www.hindawi.com

Journal of
Signal Transduction
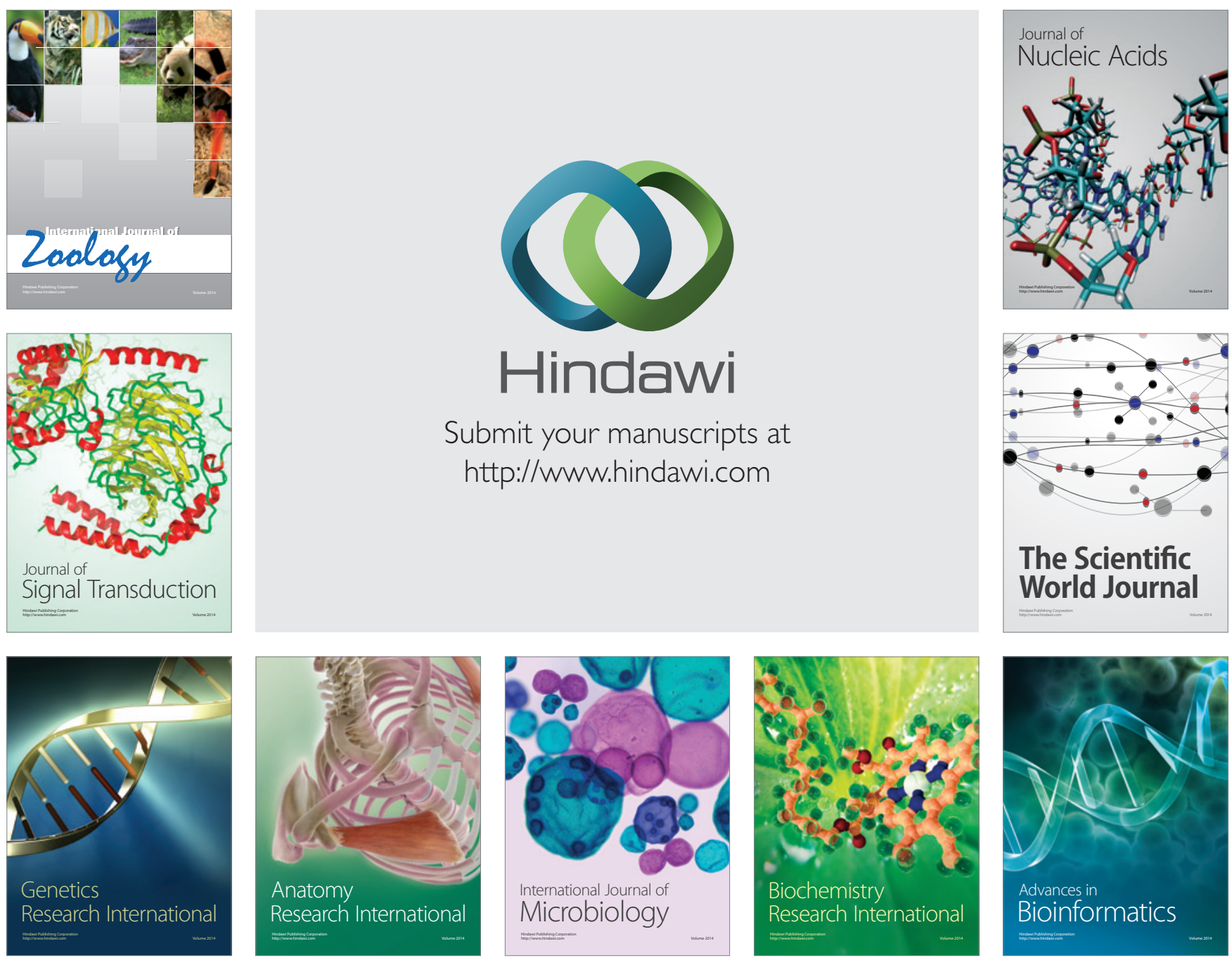

The Scientific World Journal
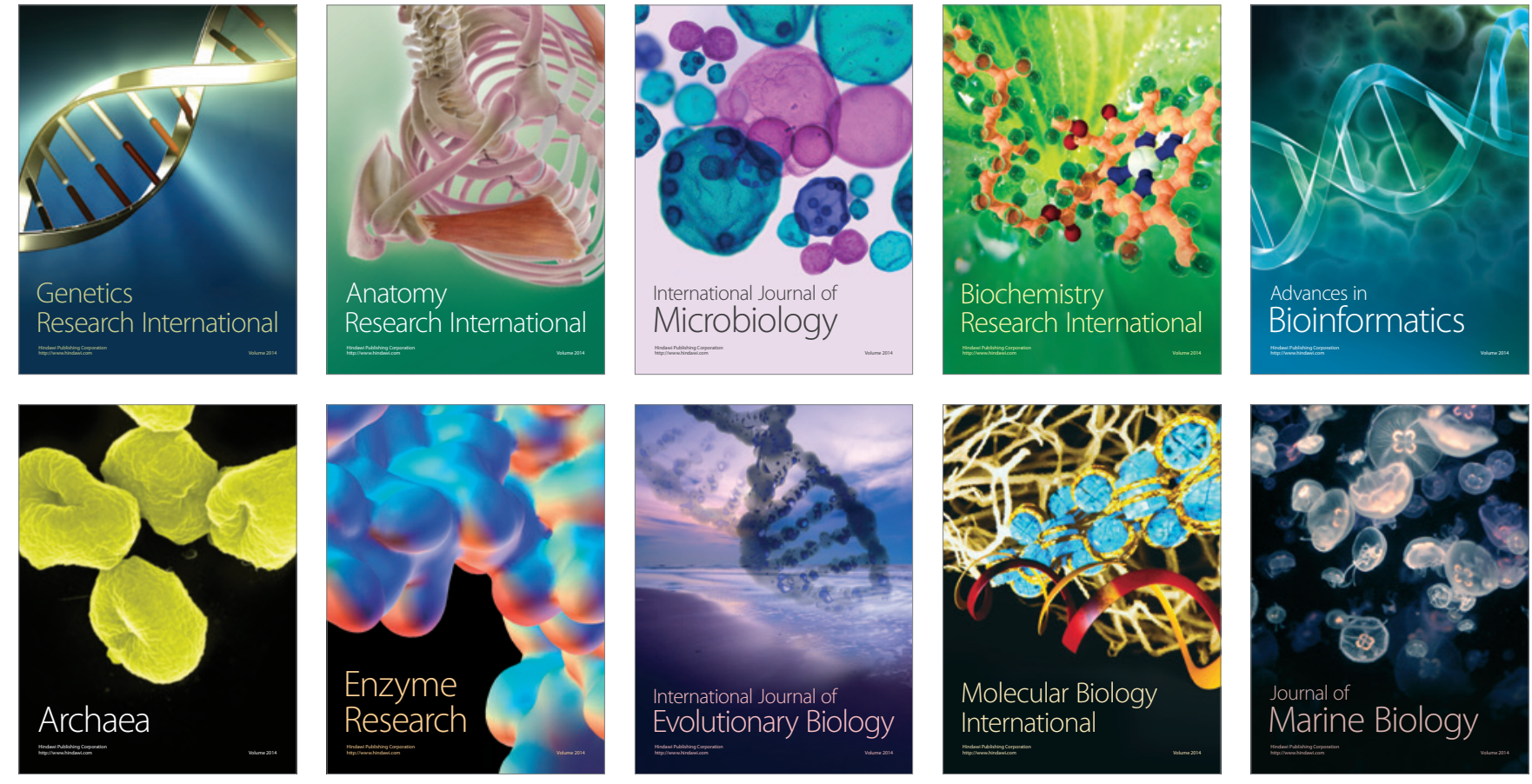